\title{
Machine Learning-Based Three-Month Outcome Prediction in Acute Ischemic Stroke: A Single Cerebrovascular-Specialty Hospital Study in South Korea
}

\author{
Dougho Park ${ }^{1}{ }^{1}$, Eunhwan Jeong ${ }^{2}$, Haejong Kim ${ }^{2}$, Hae Wook Pyun ${ }^{3}$, Haemin Kim ${ }^{4}$, Yeon-Ju Choi ${ }^{4}$, \\ Youngsoo Kim ${ }^{4}$, Suntak Jin ${ }^{4}$, Daeyoung Hong ${ }^{4}$, Dong Woo Lee ${ }^{4}$, Su Yun Lee ${ }^{2, *}$ and Mun-Chul Kim ${ }^{4, *(1)}$ \\ 1 Department of Rehabilitation Medicine, Pohang Stroke and Spine Hospital, Pohang 37659, Korea; \\ parkdougho@gmail.com \\ 2 Department of Neurology, Pohang Stroke and Spine Hospital, Pohang 37659, Korea; \\ jeh132000@hanmail.net (E.J.); openmind-2u@hanmail.net (H.K.) \\ 3 Department of Radiology, Pohang Stroke and Spine Hospital, Pohang 37659, Korea; biganhoo@naver.com \\ 4 Department of Neurosurgery, Pohang Stroke and Spine Hospital, Pohang 37659, Korea; \\ soosungzzang@hanmail.net (H.K.); ns_yeonju@naver.com (Y.J.C.); Youngsooooo@gmail.com (Y.K.); \\ zin614@gmail.com (S.J.); hongdy2000@gmail.com (D.H.); nancbr2001@gmail.com (D.W.L.) \\ * Correspondence: sunyu804@gmail.com (S.Y.L.); nsmckim@hotmail.com (M.-C.K.)
}

Citation: Park, D.; Jeong, E.; Kim, H.; Pyun, H.W.; Kim, H.; Choi, Y.-J.; Kim, Y.; Jin, S.; Hong, D.; Lee, D.W.; et al. Machine Learning-Based Three-Month Outcome Prediction in Acute Ischemic Stroke: A Single Cerebrovascular-Specialty Hospital Study in South Korea. Diagnostics 2021, 11, 1909. https://doi.org/ 10.3390/diagnostics11101909

Academic Editors: Hee-Cheol Kim and Sameer Antani

Received: 10 September 2021 Accepted: 13 October 2021

Published: 15 October 2021

Publisher's Note: MDPI stays neutral with regard to jurisdictional claims in published maps and institutional affiliations.

Copyright: (c) 2021 by the authors. Licensee MDPI, Basel, Switzerland. This article is an open access article distributed under the terms and conditions of the Creative Commons Attribution (CC BY) license (https:/ / creativecommons.org/licenses/by/ $4.0 /)$.

\begin{abstract}
Background: Functional outcomes after acute ischemic stroke are of great concern to patients and their families, as well as physicians and surgeons who make the clinical decisions. We developed machine learning (ML)-based functional outcome prediction models in acute ischemic stroke. Methods: This retrospective study used a prospective cohort database. A total of 1066 patients with acute ischemic stroke between January 2019 and March 2021 were included. Variables such as demographic factors, stroke-related factors, laboratory findings, and comorbidities were utilized at the time of admission. Five ML algorithms were applied to predict a favorable functional outcome (modified Rankin Scale 0 or 1 ) at 3 months after stroke onset. Results: Regularized logistic regression showed the best performance with an area under the receiver operating characteristic curve (AUC) of 0.86. Support vector machines represented the second-highest AUC of 0.85 with the highest F1-score of 0.86, and finally, all ML models applied achieved an AUC $>0.8$. The National Institute of Health Stroke Scale at admission and age were consistently the top two important variables for generalized logistic regression, random forest, and extreme gradient boosting models. Conclusions: ML-based functional outcome prediction models for acute ischemic stroke were validated and proven to be readily applicable and useful.
\end{abstract}

Keywords: ischemic stroke; functional outcome; algorithm; machine learning; prediction

\section{Introduction}

Stroke is a representative disease with high mortality and morbidity [1], with 30-70\% of stroke survivors reportedly remaining disabled [2]. Functional disability, directly and indirectly, affects the rest of the patient's life. It not only adversely affects the patient's return to society and work but also places a burden on family members [3]. Moreover, disability is an important cause of psychiatric complications, such as depression, which significantly reduces the long-term quality of life of patients [4]; therefore, the prognosis related to functional ability after a stroke is understandably one of the most important concerns for patients and their families. In addition, the need for predicting functional recovery is required by physicians and surgeons who need to establish long-term treatment plans [5].

From these requirements, several prediction tools using the risk scoring method have been proposed for acute ischemic stroke, which account for the majority of stroke [6]. The representative tools are the Acute Stroke Registry and Analysis of Lausanne (ASTRAL) 
score and ischemic stroke predictive risk score (ISCORE) $[7,8]$. These tools have the advantage of being simple and readily available with few variables; therefore, they can make a quick prediction with only the findings within a short time after hospitalization [9]. In previous studies, external validation results for short-term functional outcome prediction of these tools have shown an area under the receiver operating characteristic curve (AUC) range of approximately 0.8 , indicating that these tools were effective [10-12].

Meanwhile, research applying machine learning (ML) algorithms in disease diagnosis and prognosis prediction has been growing in recent years [13,14], and more specifically, studies using ML for outcome prediction in acute ischemic stroke have been published $[15,16]$. Compared with existing risk scoring methods, the ML-based prediction model has the advantage of being able to easily process a large number of samples and variables using electric health recording [17]. In addition, unlike fixed risk scoring tools, there is an evolutionary advantage that advanced ML-based models can be continuously established [18].

Based on this background, this study aimed to develop ML-based models capable of predicting the short-term favorable functional outcomes after acute ischemic stroke through the experience of a single cerebrovascular-specialty hospital in South Korea. Specifically, to share the advantageous features of existing risk scoring tools, the models were specifically developed to be applied within a short time after the stroke occurrence by using variables that can be utilized immediately after admission. Moreover, 37 variables were included by utilizing the advantages of ML modeling based on electric health recording to enable more precise and detailed outcome prediction. The predictive performances of several ML algorithms were evaluated and the best model was identified. Additionally, the variable importance related to the prediction performance was identified.

\section{Materials and Methods}

\subsection{Data Source and Patients}

This retrospective study used a prospectively enrolled cohort dataset from a single center and was conducted using the electronic health records of patients admitted to Pohang Stroke and Spine Hospital with acute ischemic stroke between January 2019 and March 2021. This study was reviewed and approved by the Institutional Review Board (Approval number: PSSH0475-2021-08-HR-016-01) and performed in compliance with the Declaration of Helsinki and the International Conference on HarmonizationGood Clinical Practice Guideline. Simultaneously, all patients included in the study were enrolled in the multicenter, prospective, and hospital-based stroke registry (Korean Stroke Registry; www.strokedb.or.kr; accessed on 25 August 2021) in South Korea. Patients or their family members provided written informed consent to agree to the utilization of the patient's non-identifiable information for research purposes when registered in the Korean Stroke Registry (Research management number: PSSH0475-201901-HR-001). We completed the final dataset for this study by applying the following exclusion criteria in order: (1) follow-up loss at 3 months, (2) having a missing value, (3) taking more than 7 days from the onset (or last normal time [LNT]) to hospitalization, (4) previous neurologic deficit (modified Rankin Scale [mRS] $\geq 2$ ), and (5) patients who died during admission (one died from pneumonia and another from hemorrhagic transformation). A flowchart of patient inclusion for this study is shown in Figure 1.

\subsection{Variables}

Variables that were initially available on admission in patients with acute ischemic stroke were used. The detailed definitions of all the contributing variables are presented in the online Supplementary Materials (Document S1).

Personal factors, such as age, sex, body mass index, and abdominal circumference, were checked. We evaluated the National Institute of Health Stroke Scale (NIHSS) at admission, stroke subtype, onset type, onset (or LNT) to arrival time, circulatory territory, involved side, and the type of acute intravenous/intraarterial treatments as stroke-related 
factors. We also investigated the initial laboratory findings and blood pressure at the time of admission. Finally, comorbidities including previous stroke or transient ischemic attack, coronary artery disease, peripheral artery diseases, hypertension, diabetes, dyslipidemia, atrial fibrillation, smoking habit, previous administration of antiplatelet/anticoagulant, and potential sources of cardiogenic embolism were checked and confirmed for the patients as well.

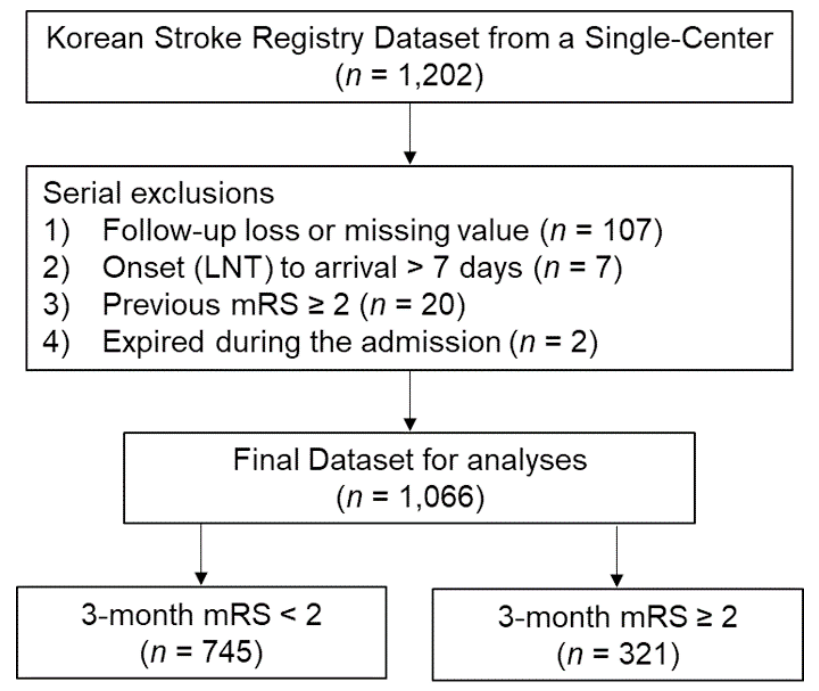

Figure 1. Flow chart of patient inclusion and exclusion. LNT, last normal time; mRS, modified Rankin Scale.

The dependent variable was defined as mRS values at 3 months after the onset of the stroke and dichotomized for prediction. Favorable outcomes were defined as mRS scores of 0 and 1 , and this group was designated as the target class, with follow-up mRS values measured at an outpatient clinic by a neurologist or neurosurgeon. In the case of not being able to visit the hospital, the mRS measurement was conducted in the form of a telephone interview with patients or their families through a structured questionnaire according to the Korean Stroke Registry guideline [19].

\subsection{Data Analysis and Machine Learning Processes}

All statistical analyses and machine learning processes were performed using $\mathrm{R}$ software 4.1.0 (R Core Team, R Foundation for Statistical Computing, Vienna, Austria). The entire code used for data analyses and the ML processes in this study, as well as the corresponding results, are available in the online Supplementary Materials (Document S2). The entire ML modeling process is summarized in Figure 2.

Comparative analyses were conducted between outcome groups with $\mathrm{mRS}<2$ or $m R S \geq 2$ at 3 months after stroke onset. Continuous variables were expressed as the mean \pm standard deviation, and an independent $t$-test was used to compare the two groups. Categorical variables were presented as frequencies and proportions, and the chi-squared (trend) test was performed for comparative analysis, with the statistical significance defined as $p<0.05$.

Serial data pre-processing was performed for ML algorithm training and validation. Variables with near-zero variances were identified and removed, with correlation coefficients evaluated to confirm the collinearity between continuous variables. After the variable selection was completed, centering and scaling for continuous variables and onehot encoding for categorical variables were conducted. The dataset was then randomly split into training and test sets at a ratio of 7:3, and the synthetic minority oversampling technique (SMOTE) and adaptive synthetic (ADASYN) sampling were then applied to the training set to deal with the imbalance in the target class. 


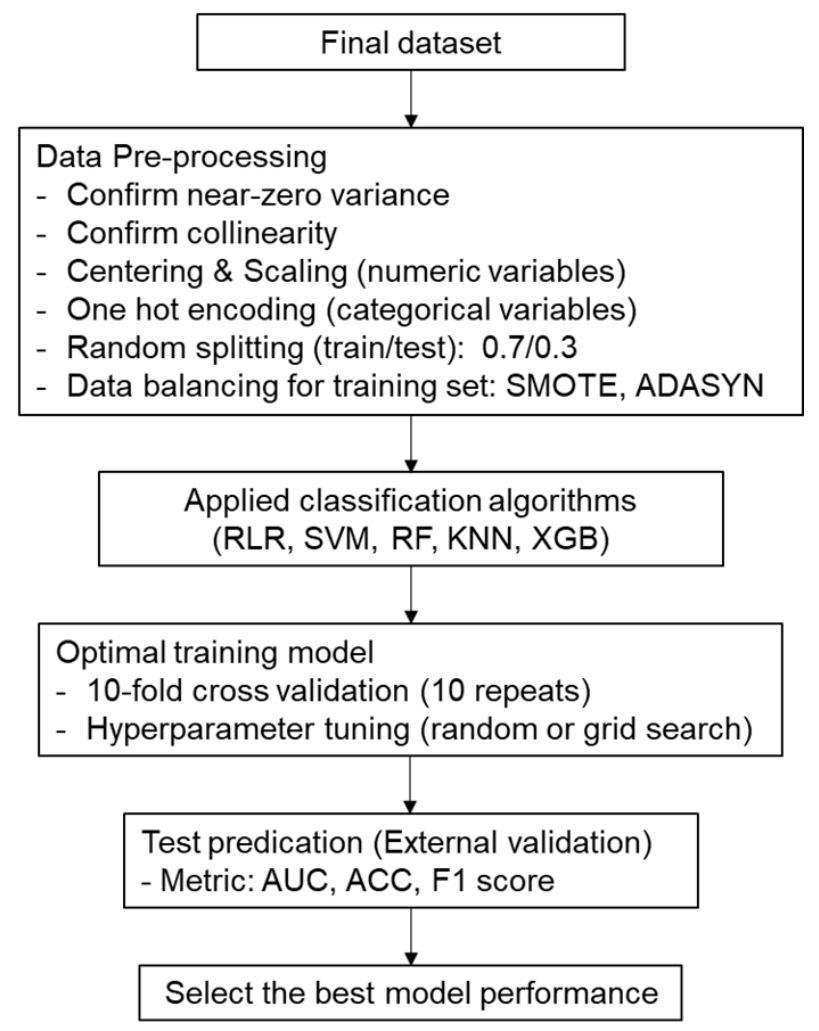

Figure 2. Entire machine learning modeling process for this study. SMOTE, synthetic minority oversampling technique; ADASYN, adaptive synthetic; RLR, regularized logistic regression; SVM, support vector machines; $\mathrm{RF}$, random forest; $\mathrm{KNN}$, k-nearest neighbors; $\mathrm{XGB}$, extreme gradient boosting; AUC, area under the receiver operating characteristic curve; ACC, accuracy.

Five ML algorithms were evaluated in this study, namely, regularized logistic regression (RLR), support vector machines (SVM), random forest (RF), k-nearest neighbors $(\mathrm{KNN})$, and extreme gradient boosting (XGB). Internal validation was performed by applying these ML algorithms to the training dataset, where pre-processing was completed. To obtain an optimal training model, 10 -fold cross-validation with 10 repetitions was performed, with both random and grid search methods applied for hyperparameter tuning. An external validation was then performed on the test dataset using the trained models. This study used AUC as the main metric and investigated the F1-score and overall accuracy to evaluate model performance. The best-performing model was then selected for each ML algorithm based on external validation results. The variable importance from ensemble algorithms (RF and XGB) and a linear model (RLR) was also determined, with the top 10 important variables from each algorithm identified.

\section{Results}

\subsection{Baseline Characteristics}

Amongst the 1066 patients analyzed, 745 (69.9\%) and 321 (30.1\%) patients had favorable and unfavorable outcomes at the 3-month follow-up, respectively.

Table 1 describes personal and stroke-related features in both outcome groups. The favorable outcome group was $65.8 \pm 11.3$ years old, which was significantly younger than that of the unfavorable outcome group, $74.4 \pm 11.4$ years old $(p<0.001)$, and the proportion of men was significantly lower $(33.7 \%$ vs. $48.6 \% ; p<0.001)$. In the favorable outcome group, NIHSS at admission was significantly lower ( $2.3 \pm 3.2$ vs. $6.3 \pm 5.9 ; p<0.001)$, and the proportion without acute intravenous/intraarterial treatments was significantly higher ( $90.3 \%$ vs. $79.1 \% ; p<0.001)$; however, in the favorable outcome group, significantly higher hemoglobin, and triglyceride levels ( $p<0.001$ and $p=0.004$, respectively), and significantly 
lower random glucose and blood urea nitrogen levels ( $p=0.028$ and $p=0.007$, respectively) were found.

Table 1. Baseline characteristics and stroke-related factors in each group.

\begin{tabular}{|c|c|c|c|}
\hline Variables & $\mathrm{mRS}<2(n=745)$ & $\mathrm{mRS} \geq 2(n=321)$ & $p$ Value \\
\hline Age, years old & $65.8 \pm 11.3$ & $74.4 \pm 11.4$ & $<0.001$ \\
\hline Male, $n(\%)$ & $251(33.7)$ & $156(48.6)$ & $<0.001$ \\
\hline Body mass index, $\mathrm{kg} / \mathrm{m}^{2}$ & $24.2 \pm 3.1$ & $23.8 \pm 3.5$ & 0.060 \\
\hline Abdominal circumference, $\mathrm{cm}$ & $85.2 \pm 9.2$ & $84.6 \pm 10.2$ & 0.310 \\
\hline NIHSS at admission & $2.3 \pm 3.2$ & $6.3 \pm 5.9$ & $<0.001$ \\
\hline Clear onset, $n(\%)$ & $605(81.2)$ & $245(76.3)$ & 0.082 \\
\hline Onset (or LNT) to arrival, day & $1.4 \pm 1.6$ & $1.2 \pm 1.5$ & 0.120 \\
\hline Stroke subtype, $n(\%)$ & & & 0.066 \\
\hline Small vessel occlusion & $289(38.8)$ & $106(33.0)$ & \\
\hline Large artery atherosclerosis & $280(37.6)$ & $143(44.5)$ & \\
\hline Cardiogenic embolism & $79(10.6)$ & $40(12.5)$ & \\
\hline Others & $97(13.0)$ & $32(10.0)$ & \\
\hline Circulation, $n(\%)$ & & & 0.097 \\
\hline Anterior & $521(69.9)$ & $244(76.0)$ & \\
\hline Posterior & $223(29.9)$ & $76(23.7)$ & \\
\hline Multiple & $1(0.1)$ & $1(0.3)$ & \\
\hline Side, $n(\%)$ & & & 0.264 \\
\hline Right & $350(47.0)$ & 153 (47.7) & \\
\hline Left & $341(45.8)$ & $136(42.4)$ & \\
\hline Bilateral & $54(7.2)$ & $32(10.0)$ & \\
\hline Acute treatment, $n(\%)$ & & & $<0.001$ \\
\hline None & $673(90.3 \%)$ & $254(79.1 \%)$ & \\
\hline Intravenous only & $13(1.7 \%)$ & $10(3.1 \%)$ & \\
\hline Intraarterial only & $38(5.1 \%)$ & $42(13.1 \%)$ & \\
\hline $\begin{array}{l}\text { Intravenous and } \\
\text { intraarterial }\end{array}$ & $21(2.8 \%)$ & $15(4.7 \%)$ & \\
\hline Systolic blood pressure, $\mathrm{mmHg}$ & $156.6 \pm 27.0$ & $159.0 \pm 26.1$ & 0.179 \\
\hline Diastolic blood pressure, $\mathrm{mmHg}$ & $87.6 \pm 15.4$ & $87.1 \pm 16.3$ & 0.609 \\
\hline Hemoglobin, g/dl & $14.1 \pm 1.7$ & $13.4 \pm 1.9$ & $<0.001$ \\
\hline Platelet, $10^{3} / \mu \mathrm{L}$ & $238.3 \pm 66.1$ & $231.5 \pm 68.6$ & 0.125 \\
\hline Total cholesterol, $\mathrm{mg} / \mathrm{dl}$ & $191.8 \pm 46.5$ & $188.6 \pm 48.4$ & 0.305 \\
\hline Triglyceride, $\mathrm{mg} / \mathrm{dl}$ & $166.4 \pm 131.4$ & $141.2 \pm 127.2$ & 0.004 \\
\hline High density lipoprotein, $\mathrm{mg} / \mathrm{dl}$ & $49.3 \pm 12.2$ & $48.1 \pm 11.6$ & 0.135 \\
\hline Low density lipoprotein, mg/dl & $109.4 \pm 40.6$ & $113.0 \pm 42.6$ & 0.189 \\
\hline Random glucose, mg/dl & $140.8 \pm 57.1$ & $149.7 \pm 62.6$ & 0.028 \\
\hline $\mathrm{HbA} 1 \mathrm{c}, \%$ & $6.2 \pm 1.2$ & $6.4 \pm 1.4$ & 0.077 \\
\hline Blood urea nitrogen, $\mathrm{mg} / \mathrm{dl}$ & $16.4 \pm 5.4$ & $17.7 \pm 7.6$ & 0.007 \\
\hline Creatinine, $\mathrm{mg} / \mathrm{dl}$ & $0.9 \pm 0.3$ & $0.9 \pm 0.4$ & 0.389 \\
\hline
\end{tabular}

mRS, modified Rankin Scale; NIHSS, National Institutes of Health Stroke; LNT, last normal time.

Table 2 presents the underlying risk factors for both outcome groups. It was found that the ratio of current smokers was significantly higher in the favorable outcome group $(p=0.002)$, whilst atrial fibrillation was significantly higher in the unfavorable outcome group $(p=0.009)$. There were no significant differences in other risk factors found between the two groups.

\subsection{Data Pre-Processing}

The following variables were removed that showed near-zero variance: previous transient ischemic attack, previous peripheral artery diseases, previous cancer, previous administration of anticoagulant, and high and medium risks of potential sources of cardiogenic embolism. It should also be noted that none of the continuous variables showed any collinearity. 
Table 2. Underlying risk factors in each group.

\begin{tabular}{|c|c|c|c|}
\hline Variables & $\mathrm{mRS}<2(n=745)$ & $\mathrm{mRS} \geq 2(n=321)$ & $p$ Value \\
\hline Previous stroke, $n(\%)$ & & & 0.785 \\
\hline None & $640(85.9)$ & $270(84.1)$ & \\
\hline Hemorrhagic & $17(2.3)$ & $9(2.8)$ & \\
\hline Ischemic & $85(11.4)$ & $40(12.5)$ & \\
\hline Mixed & $2(0.3)$ & $2(0.6)$ & \\
\hline Unknown & $1(0.1)$ & $0(0.0)$ & \\
\hline Previous TIA, $n(\%)$ & $14(1.9)$ & $4(1.2)$ & 0.633 \\
\hline Previous PAD, $n(\%)$ & $4(0.5)$ & $3(0.9)$ & 0.746 \\
\hline Previous CAD, $n(\%)$ & $72(9.7)$ & $38(11.8)$ & 0.337 \\
\hline Previous cancer, $n(\%)$ & $9(1.2)$ & $5(1.6)$ & 0.868 \\
\hline Previous hypertension, $n(\%)$ & & & 0.224 \\
\hline None & $293(39.3)$ & $109(34.0)$ & \\
\hline Known & $395(53.0)$ & $188(58.6)$ & \\
\hline Diagnosed at admission & $57(7.7)$ & $24(7.5)$ & \\
\hline Previous diabetes, $n(\%)$ & & & 0.087 \\
\hline None & $556(74.6)$ & $223(69.5)$ & \\
\hline Known & $159(21.3)$ & $88(27.4)$ & \\
\hline Diagnosed at admission & $30(4.0)$ & $10(3.1)$ & \\
\hline Previous dyslipidemia, $n(\%)$ & & & 0.650 \\
\hline None & $278(37.3)$ & $122(38.0)$ & \\
\hline Known & $222(29.8)$ & $87(27.1)$ & \\
\hline Diagnosed at admission & $245(32.9)$ & $112(34.9)$ & \\
\hline Smoking, $n(\%)$ & & & 0.002 \\
\hline None & $499(67.0)$ & $252(78.5)$ & \\
\hline Current & $242(32.5)$ & $69(21.5)$ & \\
\hline Quit $\geq 5$ years & $2(0.3)$ & $0(0.0)$ & \\
\hline Quit $<5$ years & $2(0.3)$ & $0(0.0)$ & \\
\hline Previous AF, $n(\%)$ & & & 0.009 \\
\hline None & $658(88.3)$ & $261(81.3)$ & \\
\hline Known & $41(5.5)$ & $30(9.3)$ & \\
\hline Diagnosed at admission & $46(6.2)$ & $30(9.3)$ & \\
\hline Previous antiplatelet, $n(\%)$ & $191(25.6)$ & $81(25.2)$ & 0.950 \\
\hline Previous anticoagulation, $n(\%)$ & $19(2.6)$ & $14(4.4)$ & 0.170 \\
\hline PSCE-high risk, $n(\%)$ & $20(2.7)$ & $8(2.5)$ & $>0.999$ \\
\hline PSCE-medium risk, $n(\%)$ & $34(4.6)$ & $17(5.3)$ & 0.721 \\
\hline
\end{tabular}

mRS, modified Rankin Scale; TIA, transient ischemic attack; PAD, peripheral artery disease; CAD, coronary artery disease; AF, atrial fibrillation; PSCE, potential sources of cardiogenic embolism.

After random splitting, the training and test datasets were divided into 769 and 297 samples, respectively, with the proportions of favorable and unfavorable outcome groups in the training dataset being 537 and 232, respectively. After applying SMOTE, the ratios were 537 and 464, respectively, and after applying ADASYN, the ratios were 537 and 555 , respectively. In the test dataset, the two outcome groups had ratios of 208 and 89.

\subsection{Performances of Machine Learning Algorithms}

RLR showed the best performance among all ML algorithms utilized with an AUC of 0.86 (95\% confidence interval [CI], 0.82-0.90). Meanwhile, SVM showed the second-highest AUC of 0.85 (95\% CI, 0.81-0.89) and recorded the highest overall accuracy and F1-score ( 0.80 and 0.86, respectively). RF and KNN showed an AUC value of 0.82 (95\% CI, 0.77-0.87), and XGB was 0.81 (95\% CI, 0.76-0.86) (Table 3 and Figure 3). The confusion matrix for each ML algorithm is presented in Supplementary Table S1.

Among the optimal training models of the best test prediction result for each ML algorithm, SVM, XGB, and KNN showed the best performance when target class balancing was not performed. Contrarily, the best-performed model was generated in RLR and RF when SMOTE and ADASYN were applied, respectively. Supplementary Table S2 shows the balancing method and hyperparameter tuning results for the best model of each ML algorithm. 
Table 3. Model performances of each machine learning model on the test dataset.

\begin{tabular}{cccccc}
\hline Metric & RLR & SVM & RF & KNN & XGB \\
\hline AUC & 0.86 & 0.85 & 0.82 & 0.82 & 0.81 \\
$(95 \%$ CI $)$ & $(0.82-0.90)$ & $(0.81-0.89)$ & $(0.77-0.87)$ & $(0.77-0.87)$ & $(0.76-0.86)$ \\
\hline F1 & 0.84 & 0.86 & 0.85 & 0.84 & 0.85 \\
\hline Accuracy & 0.78 & 0.80 & 0.77 & 0.73 & 0.77 \\
$(95 \%$ CI $)$ & $(0.73-0.83)$ & $(0.75-0.85)$ & $(0.72-0.82)$ & $(0.67-0.78)$ & $(0.72-0.82)$ \\
\hline $\begin{array}{c}\text { Sensitivity } \\
(95 \% \text { CI) }\end{array}$ & 0.83 & 0.90 & 0.89 & 0.99 & 0.89 \\
\hline $\begin{array}{c}\text { Specificity } \\
(95 \% \text { CI) }\end{array}$ & $0.77-0.87)$ & $(0.86-0.94)$ & $(0.83-0.92)$ & $(0.97-1.00)$ & $(0.84-0.93)$ \\
\hline $\begin{array}{c}\text { Precision } \\
(95 \% \text { CI) }\end{array}$ & $(0.81-0.90)$ & $(0.77-0.87)$ & $(0.76-0.86)$ & $(0.67-0.77)$ & $(0.75-0.85)$ \\
\hline NPV & 0.63 & 0.71 & 0.53 & 0.10 & 0.50 \\
$(95 \%$ CI $)$ & $(0.53-0.72)$ & $(0.60-0.81)$ & $(0.55-0.76)$ & $(0.60-0.98)$ & $(0.54-0.76)$ \\
\hline
\end{tabular}

RLR, regularized logistic regression; SVM, support vector machines; RF, random forest; KNN, k-nearest neighbors, $\mathrm{XGB}$, extreme gradient boosting; $\mathrm{AUC}$, area under the receiver operating characteristic curve; $\mathrm{ACC}$, accuracy; $\mathrm{NPV}$, negative predictive value.

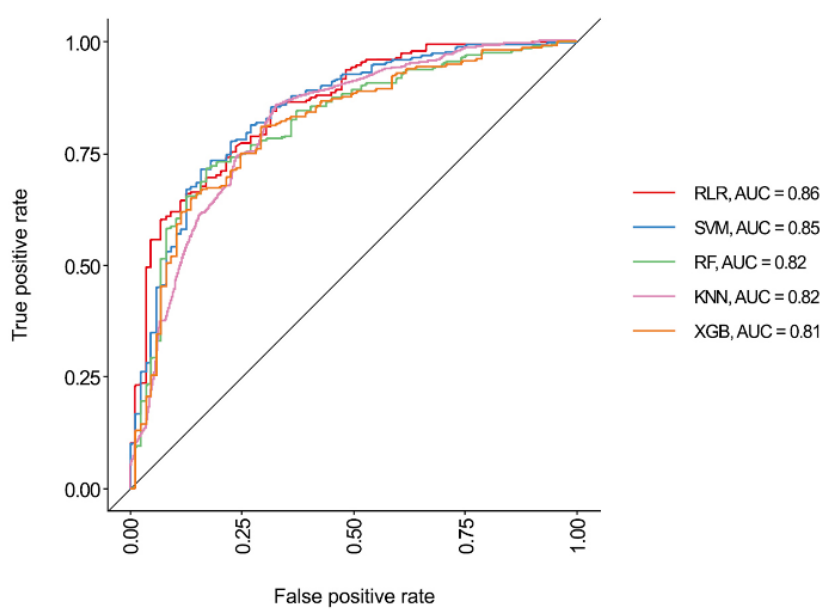

(a)

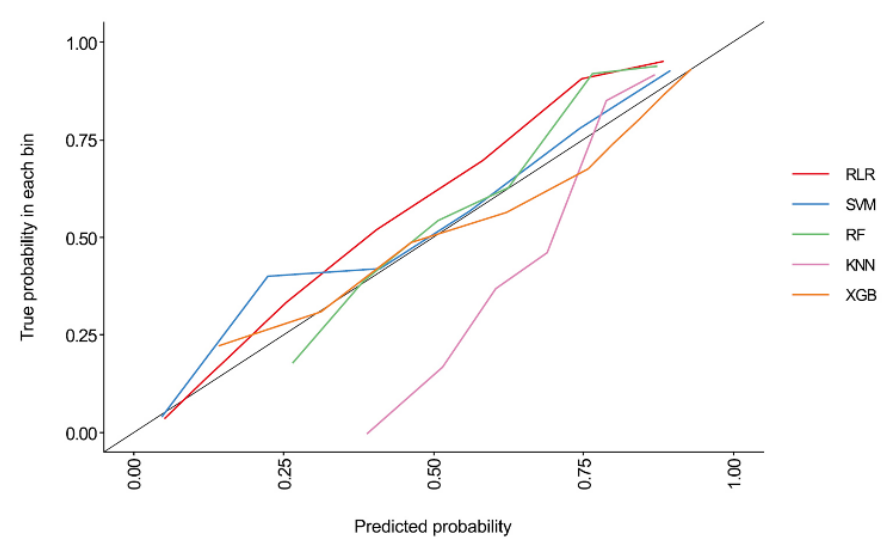

(b)

Figure 3. Results of external validation of each machine learning algorithm. (a) Receiver operating characteristic curves and (b) calibration plots are represented. The regularized logistic regression model showed the best performance with an AUC of 0.86 (red line). Overall, all the ML models showed AUC > 0.8. AUC, area under the receiver operating characteristic curve; RLR, regularized logistic regression; SVM, support vector machines; RF, random forest; KNN, k-nearest neighbors; $\mathrm{XGB}$, extreme gradient boosting.

\subsection{Variable Importance}

For RLR, NIHSS was the most important variable for model performance, followed by age and hemoglobin. Both RF and XGB represented the same top three results of variable importance; the most important variable for the prediction performance was the NIHSS score at admission, followed by age and time to arrival. NHISS at admission and age were also the two most important variables in the three ML models. Finally, random glucose, hemoglobin, and triglyceride levels were identified as the top ten important variables in all three models (Figure 4). 
Regularized logistic regression

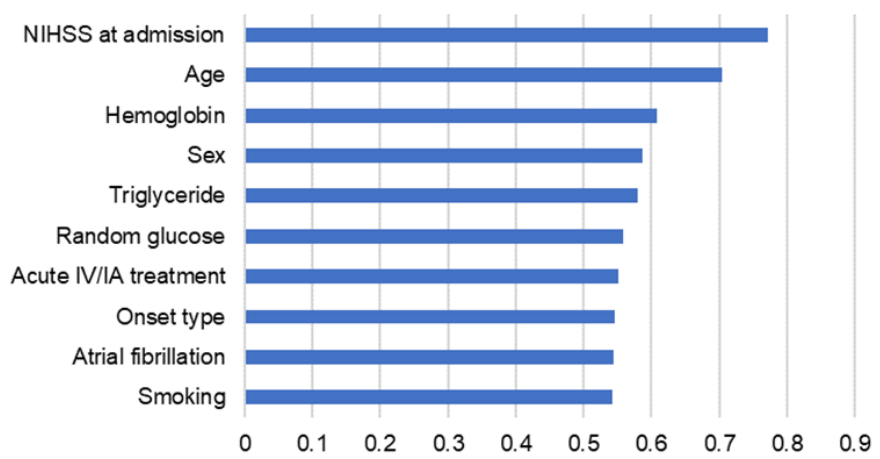

(a)

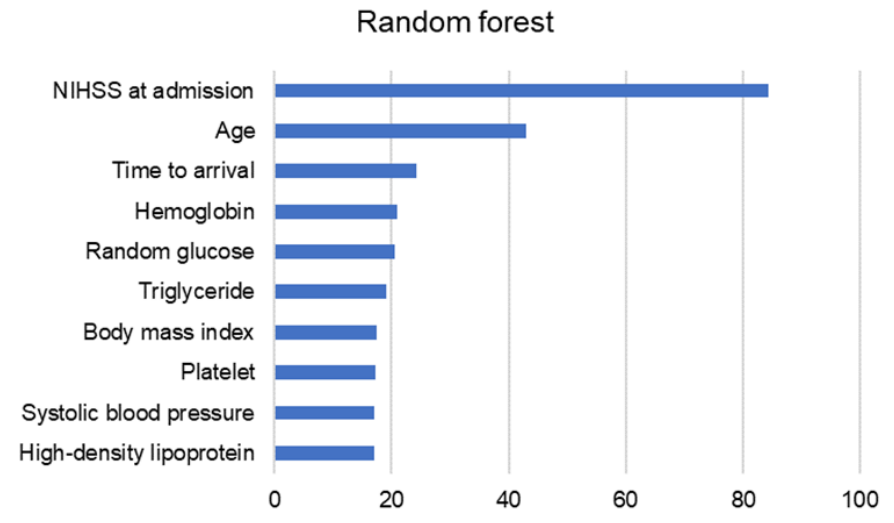

(b)

Extreme gradient boosting

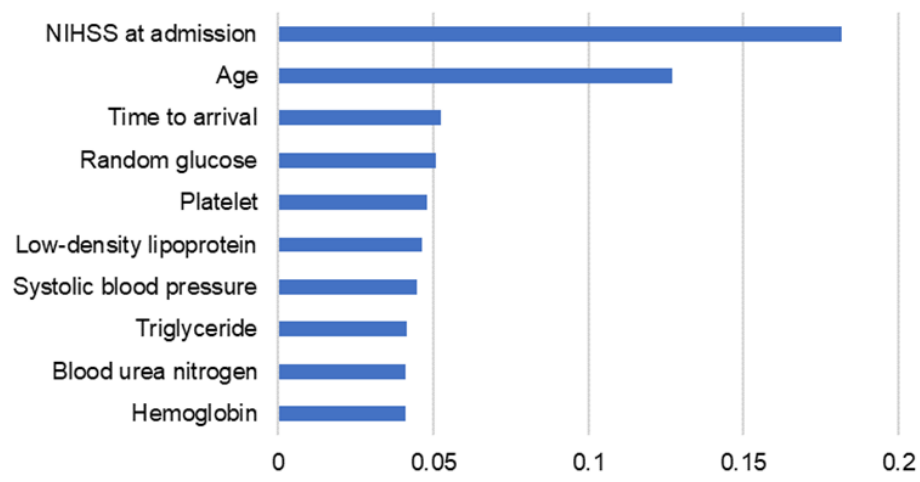

(c)

Figure 4. Top ten important variables in (a) regularized logistic regression, (b) random forest, and (c) extreme gradient boosting models. The top two important features were consistent in all three models; NIHSS at admission, followed by age. Additionally, random glucose, hemoglobin, and triglyceride were also included in the top ten important variables in all three models. NIHSS, National Institute of Health Stroke Scale; IV, intravenous; IA, intraarterial.

\section{Discussion}

This study demonstrated models that predict the short-term functional prognosis of patients with acute ischemic stroke using ML algorithms. All ML algorithms utilized showed validated results with AUC $>0.8$. In particular, the proposed models were established based on initial evaluation and examination findings at the time of admission, which has the advantage of being able to predict a favorable outcome within a short time after hospitalization. Moreover, this feature can be useful to physicians and surgeons in making clinical decisions or informing patients and their families.

The proposed ML models' performance was either similar to or slightly better than existing risk scoring tools such as ASTRAL and ISCORE; nevertheless, it is difficult to conclude that our ML models using more variables are a much-improved prediction tool. The reason for this finding can be attributed to the following. The first is the difference in outcome definition. Unlike both ASTRAL and ISCORE, where mRS 0-2 was defined as a good outcome, only mRS 0 and 1 were defined as favorable outcomes in this study. According to mRS [20], if based on dependency, grade 2 or less can be viewed as a favorable outcome; however, when a narrow favorable outcome criterion was applied, we considered the absence of disability and maintenance of usual daily activities; therefore, it is difficult to compare these two risk scoring tools directly with the proposed ML models because of the different target outcome definitions. The second reason can be inferred from the results of the variable importance. The ASTRAL score is based on six contributing factors: age, NIHSS score, time delay, visual field defect, glucose level, and level of consciousness [7]. 
ISCORE is calculated based on age, sex, preadmission functionality, cancer, atrial fibrillation, congestive heart failure, renal function, stroke subtype, glucose level, and stroke severity [8]. There is a significant overlap between the contributing factors of these two tools and the variables with high importance in our ML models. Several more variables were utilized compared to the existing risk scoring tools by taking advantage of ML-based data processing; however, it was found that they shared a similar critical variables list.

There have been previous studies on the ML-based predictions of functional outcomes in acute ischemic stroke. Heo et al. [11] predicted a favorable functional outcome at the 3-month follow-up. They designated mRS 0-2 as the target class and directly compared the predictive power of their ML models to the ASTRAL score. Hence, the deep neural network recorded a significantly higher AUC than the ASTRAL score; however, there was no significant difference in the AUC between the ASTRAL score and RF and logistic regression. Alaka et al. [21] also presented ML-based models for predicting the 3-month functional outcome by targeting $\mathrm{mRS}>2$ in ischemic stroke. The external validation results in their study were inferior to our study, with an AUC range of 0.66 to 0.71 . This difference is thought to be due to our model using more sample sizes and variables than theirs. Jang et al. [22] defined mRS $>1$ at 3 months in acute ischemic stroke as a bad outcome and performed an analysis based on the same outcome class classification as ours. Among the ML models presented by them, XGB, RF, and SVM showed the highest AUC value of 0.84 ; our RLR and SVM models slightly outperformed the other models with higher AUC values.

Among the ML algorithms we applied, RLR showed the best performance. Regularization lowers the weight of the parameter to reduce the complexity of the dataset and prevent overfitting [23,24]. Thus, a linear model sometimes outperforms ensemble algorithms, such as RF and XGB $[25,26]$. We were able to optimally train our best model by applying L1 regularization, which avoids overfitting by increasing the sparsity [27]. Based on this, it is inferred that our prediction model performed better on the dataset with lower complexity in a relatively limited manner. Consequently, our linear model showed better prediction performance than the other ML algorithms in this study.

The target class in the dataset is slightly imbalanced. We applied the SMOTE and ADASYN methods to address this problem. Both SMOTE and ADASYN are KNN-based up-sampling methods [28,29]. We obtained the best results by applying SMOTE to our best model, RLR. RF was able to obtain optimal results by applying ADASYN; however, in SVM and KNN, the training model without balancing showed better results, which is thought to be because the balancing of the training set caused overfitting. XGB can avoid overfitting even with balancing methods; however, the AUC was slightly higher when we did not implement balancing methods. Consequently, to create an ideal ML-based prediction model, it is necessary to confirm the results derived from the original dataset as well as the results with target class balancing. Additionally, it is necessary to consider overfitting caused by up-sampling.

Our ML models showed relatively low specificity, which is thought to be because our target was the majority class. We chose AUC as the main metric because it is not affected by the majority or minority of the target class [30,31]. In contrast, we should interpret the overall accuracy with caution because majority class predictivity may be overestimated in imbalanced data [32]. In fact, we selected the RLR model as the best model because it showed the test prediction result with the highest AUC and the most balanced sensitivity and specificity values among the investigated models.

There were several limitations to this study. First, mRS was used as an indicator of functional outcome, which had the advantage of an intuitive understanding of the functional level; however, it can be challenging to obtain a detailed reflection of the various neurologic symptoms observed after ischemic stroke, such as dysarthria-clumsy hand, ataxic hemiparesis, and pure sensory stroke. In particular, there may be a discrepancy between the measured functional score and the discomfort of the symptoms felt by the patient in these subtypes [33]; therefore, it was believed that a better model could be presented if 
more specific clinical data were added. Second, it was a single-center study. Therefore, an integrated, multicenter study is required to generalize this study's result and may present better results. Finally, relatively broad inclusion criteria were also applied to develop a generally applicable model for acute ischemic stroke, which may have contributed to the bias.

\section{Conclusions}

This study demonstrated that ML-based models early and effectively predicted favorable functional outcomes at 3 months after acute ischemic stroke. All ML-based prediction models in this study showed validated results, with an AUC $>0.8$. In particular, RLR showed the best performance, with SVM showing promising results as well. Both models exhibited similar or slightly better performance than existing risk scoring tools or previously proposed ML-based prediction models; moreover, they are useful because they are readily applicable, informative to patients and families, and support clinical decision-making.

Supplementary Materials: The following are available online at https:/ / www.mdpi.com/article/10 .3390/diagnostics11101909/s1, Table S1: Optimal training model of each machine learning algorithm, Table S2: Confusion matrix, Document S1: Variable definitions and measurements, Document S2: R Markdown.

Author Contributions: Conceptualization and design: D.P., D.H., S.Y.L. and M.-C.K. Data curation: D.P., E.J., H.K. (Haejong Kim) and S.Y.L. Investigation: H.W.P., H.K. (Haemin Kim), Y.-J.C., Y.K., S.J. and D.W.L. Formal analysis: D.P. Validation: D.P., E.J., H.K. (Haejong Kim) and H.W.P. Writingoriginal draft: D.P., D.H., S.Y.L. and M.-C.K. Writing-review and editing: D.P., H.K. (Haemin Kim), Y.-J.C., Y.K., S.J., D.W.L., S.Y.L. and M.-C.K. Visualization: D.P. All authors have read and agreed to the published version of the manuscript.

Funding: This research received no external funding.

Institutional Review Board Statement: This study was reviewed and approved by the Institutional Review Board of Pohang Stroke and Spine Hospital (Approval number: PSSH0475-2021-08-HR-01601; Approval date: 24 August 2021) and performed in compliance with the Declaration of Helsinki and the International Conference on Harmonization-Good Clinical Practice Guideline.

Informed Consent Statement: Informed consent for this publication was waived by the Institutional Review Board of Pohang Stroke and Spine Hospital due to the retrospective nature of the study design. All patients included in this study provided written informed consent and were enrolled in the Korean Stroke Registry (Research management number: PSSH0475-201901-HR-001).

Data Availability Statement: Data are not publicly available because of privacy and ethical restrictions on the data sharing regulation of the Korean Stroke Registry. Only authorized researchers can access the dataset used in this study (www.stokedb.or.kr; accessed on 25 August 2021). The data analyses and machine learning results for this study are available in the online supplementary content.

Acknowledgments: The authors thank the hospital's stroke task force team, Hyun Jin Kong, and Yoo Jeong Jo.

Conflicts of Interest: The authors declare no conflict of interest.

\section{References}

1. Donkor, E.S. Stroke in the 21st Century: A Snapshot of the Burden, Epidemiology, and Quality of Life. Stroke Res. Treat. 2018, 2018, 3238165. [CrossRef] [PubMed]

2. Yang, Y.; Shi, Y.Z.; Zhang, N.; Wang, S.; Ungvari, G.S.; Ng, C.H.; Wang, Y.L.; Zhao, X.Q.; Wang, Y.J.; Wang, C.X.; et al. The Disability Rate of 5-Year Post-Stroke and Its Correlation Factors: A National Survey in China. PLoS ONE 2016, 11, e0165341. [CrossRef]

3. Kavga, A.; Kalemikerakis, I.; Faros, A.; Milaka, M.; Tsekoura, D.; Skoulatou, M.; Tsatsou, I.; Govina, O. The Effects of Patients' and Caregivers' Characteristics on the Burden of Families Caring for Stroke Survivors. Int. J. Environ. Res. Public Health 2021, 18, 7298. [CrossRef]

4. Paolucci, S.; Iosa, M.; Coiro, P.; Venturiero, V.; Savo, A.; De Angelis, D.; Morone, G. Post-stroke Depression Increases Disability More Than 15\% in Ischemic Stroke Survivors: A Case-Control Study. Front. Neurol. 2019, 10, 926. [CrossRef] [PubMed] 
5. Hung, M.C.; Hsieh, C.L.; Hwang, J.S.; Jeng, J.S.; Wang, J.D. Estimation of the long-term care needs of stroke patients by integrating functional disability and survival. PLoS ONE 2013, 8, e75605. [CrossRef]

6. Matsumoto, K.; Nohara, Y.; Soejima, H.; Yonehara, T.; Nakashima, N.; Kamouchi, M. Stroke Prognostic Scores and Data-Driven Prediction of Clinical Outcomes After Acute Ischemic Stroke. Stroke 2020, 51, 1477-1483. [CrossRef]

7. Ntaios, G.; Faouzi, M.; Ferrari, J.; Lang, W.; Vemmos, K.; Michel, P. An integer-based score to predict functional outcome in acute ischemic stroke: The ASTRAL score. Neurology 2012, 78, 1916-1922. [CrossRef] [PubMed]

8. Saposnik, G.; Kapral, M.K.; Liu, Y.; Hall, R.; O'Donnell, M.; Raptis, S.; Tu, J.V.; Mamdani, M.; Austin, P.C.; Investigators of the Registry of the Canadian Stroke Network; et al. IScore: A risk score to predict death early after hospitalization for an acute ischemic stroke. Circulation 2011, 123, 739-749. [CrossRef]

9. Drozdowska, B.A.; Singh, S.; Quinn, T.J. Thinking About the Future: A Review of Prognostic Scales Used in Acute Stroke. Front. Neurol. 2019, 10, 274. [CrossRef]

10. Wang, W.Y.; Sang, W.W.; Jin, D.; Yan, S.M.; Hong, Y.; Zhang, H.; Yang, X. The Prognostic Value of the iScore, the PLAN Score, and the ASTRAL Score in Acute Ischemic Stroke. J. Stroke Cerebrovasc. Dis. 2017, 26, 1233-1238. [CrossRef]

11. Heo, J.; Yoon, J.G.; Park, H.; Kim, Y.D.; Nam, H.S.; Heo, J.H. Machine Learning-Based Model for Prediction of Outcomes in Acute Stroke. Stroke 2019, 50, 1263-1265. [CrossRef]

12. Liu, G.; Ntaios, G.; Zheng, H.; Wang, Y.; Michel, P.; Wang, D.Z.; Fang, J.; Papavasileiou, V.; Liu, L.; Dong, K.; et al. External validation of the ASTRAL score to predict 3- and 12-month functional outcome in the China National Stroke Registry. Stroke 2013, 44, 1443-1445. [CrossRef] [PubMed]

13. Liew, B.X.W.; Peolsson, A.; Rugamer, D.; Wibault, J.; Lofgren, H.; Dedering, A.; Zsigmond, P.; Falla, D. Clinical predictive modelling of post-surgical recovery in individuals with cervical radiculopathy: A machine learning approach. Sci. Rep. 2020, 10, 16782. [CrossRef]

14. Krittanawong, C.; Virk, H.U.H.; Bangalore, S.; Wang, Z.; Johnson, K.W.; Pinotti, R.; Zhang, H.; Kaplin, S.; Narasimhan, B.; Kitai, T.; et al. Machine learning prediction in cardiovascular diseases: A meta-analysis. Sci. Rep. 2020, 10, 16057. [CrossRef]

15. Scrutinio, D.; Ricciardi, C.; Donisi, L.; Losavio, E.; Battista, P.; Guida, P.; Cesarelli, M.; Pagano, G.; D’Addio, G. Machine learning to predict mortality after rehabilitation among patients with severe stroke. Sci. Rep. 2020, 10, 20127. [CrossRef] [PubMed]

16. Xu, Y.; Yang, X.; Huang, H.; Peng, C.; Ge, Y.; Wu, H.; Wang, J.; Xiong, G.; Yi, Y. Extreme Gradient Boosting Model Has a Better Performance in Predicting the Risk of 90-Day Readmissions in Patients with Ischaemic Stroke. J. Stroke Cerebrovasc. Dis. 2019, 28, 104441. [CrossRef] [PubMed]

17. Kim, J.O.R.; Jeong, Y.S.; Kim, J.H.; Lee, J.W.; Park, D.; Kim, H.S. Machine Learning-Based Cardiovascular Disease Prediction Model: A Cohort Study on the Korean National Health Insurance Service Health Screening Database. Diagnostics 2021, 11, 943. [CrossRef]

18. Liebeskind, D.S.; Scalzo, F. Editorial: Machine Learning and Decision Support in Stroke. Front. Neurol. 2020, 11, 486. [CrossRef]

19. Korean Stroke Society. Korean Stroke Registry Guideline. Available online: http://www.strokedb.or.kr/bbs/index.asp?bbs_ code=open_material (accessed on 3 August 2021).

20. Quinn, T.J.; Dawson, J.; Walters, M. John Rankin; his life, legacy and the 50th anniversary of the Rankin Stroke Scale. Scott. Med. J. 2008, 53, 44-47. [CrossRef]

21. Alaka, S.A.; Menon, B.K.; Brobbey, A.; Williamson, T.; Goyal, M.; Demchuk, A.M.; Hill, M.D.; Sajobi, T.T. Functional Outcome Prediction in Ischemic Stroke: A Comparison of Machine Learning Algorithms and Regression Models. Front. Neurol. 2020, 11, 889. [CrossRef]

22. Jang, S.K.; Chang, J.Y.; Lee, J.S.; Lee, E.J.; Kim, Y.H.; Han, J.H.; Chang, D.I.; Cho, H.J.; Cha, J.K.; Yu, K.H.; et al. Reliability and Clinical Utility of Machine Learning to Predict Stroke Prognosis: Comparison with Logistic Regression. J. Stroke 2020, 22, $403-406$. [CrossRef]

23. Friedman, J.; Hastie, T.; Tibshirani, R. Regularization Paths for Generalized Linear Models via Coordinate Descent. J. Stat. Softw. 2010, 33, 1-22. [CrossRef] [PubMed]

24. Chen, D.W.; Miao, R.; Deng, Z.Y.; Lu, Y.Y.; Liang, Y.; Huang, L. Sparse Logistic Regression With L 1/2 Penalty for Emotion Recognition in Electroencephalography Classification. Front. Neuroinform. 2020, 14, 29. [CrossRef] [PubMed]

25. Li, W.; Liu, H.; Yang, P.; Xie, W. Supporting Regularized Logistic Regression Privately and Efficiently. PLoS ONE 2016, 11, e0156479. [CrossRef] [PubMed]

26. Stiglic, G.; Povalej Brzan, P.; Fijacko, N.; Wang, F.; Delibasic, B.; Kalousis, A.; Obradovic, Z. Comprehensible Predictive Modeling Using Regularized Logistic Regression and Comorbidity Based Features. PLoS ONE 2015, 10, e0144439. [CrossRef] [PubMed]

27. Reid, S.; Tibshirani, R. Regularization Paths for Conditional Logistic Regression: The clogitL1 Package. J. Stat. Softw. 2014, 58, 12. [CrossRef]

28. Blagus, R.; Lusa, L. SMOTE for high-dimensional class-imbalanced data. BMC Bioinform. 2013, 14. [CrossRef]

29. Haibo, H.; Yang, B.; Garcia, E.A.; Shutao, L. ADASYN: Adaptive synthetic sampling approach for imbalanced learning. In Proceedings of the 2008 IEEE International Joint Conference on Neural Networks (IEEE World Congress on Computational Intelligence), Hong Kong, China, 1-8 June 2008; pp. 1322-1328. 
30. Bradley, A.P. The use of the area under the ROC curve in the evaluation of machine learning algorithms. Pattern Recognit. 1997, 30, 1145-1159. [CrossRef]

31. Rosset, S. Model selection via the AUC. In Proceedings of the Twenty-First International Conference on Machine Learning-ICML, Banff Alberta, Canada, 4 July 2004.

32. Luque, A.; Carrasco, A.; Martín, A.; de las Heras, A. The impact of class imbalance in classification performance metrics based on the binary confusion matrix. Pattern Recognit. 2019, 91, 216-231. [CrossRef]

33. Arboix, A.; Garcia-Eroles, L.; Comes, E.; Oliveres, M.; Balcells, M.; Pacheco, G.; Targa, C. Predicting spontaneous early neurological recovery after acute ischemic stroke. Eur. J. Neurol. 2003, 10, 429-435. [CrossRef] 